\title{
ERRATUM
}

\section{New Insights in Extragalactic Magnetic Fields - ERRATUM}

\section{Luigina Feretti, Federica Govoni, George Heald, Lawrence Rudnick and Melanie Johnston-Hollit}

doi: 10.1017/S1743921319004423, Published online by Cambridge University Press 03 March, 2020

In the publication of the Astronomy in Focus series, volume XXX, a number of pieces of supplementary material were omitted, including for Focus Meeting 8.

The missing supplementary material has now been uploaded and assigned to this introductory article.

\section{References}

Feretti, L., Govoni, F., Heald, G., Rudnick, L., \& Johnston-Hollitt, M. (2018). New Insights in Extragalactic Magnetic Fields. Proceedings of the International Astronomical Union, 14(A30), 287-290. doi: 10.1017/S1743921319004423 